\title{
Research on the Reading Application of Smart TV
}

\author{
Jie Fan Xin Xie Yongzhi Lou \\ Beijing Union University, Beijing, 100101, China
}

\begin{abstract}
With the development of Chinese economy, the quality of population has generally improved. More and more people pay attention to the concept of sustainable development of family education. The two-way parenting theory is more suitable for modern social requirements. Here two-way parenting theory is the breakthrough parenting theory. The people who hold the theory argue against the fragmentation of knowledge. The theory provides a nurturing system that responds to each other. It combines ideas, specifications, and methods perfectly. Based on the breakthrough parenting theory which is full of love, all family members constructed a new educational bookstore together. At present we mainly discuss the application model of interactive design of electronic reading on TV. First, based on the desire for a better life, all the people are looking forward to a better education. For example, we construct a virtual chat environment for interactive reading of artistic education in the future through the application of television reading about the theme of tea culture. Secondly, a new sense of immersion in the interior environment was created. At this time the concept of low-carbon life will be formed in the brains of the people though the artistic information resources. Thirdly, it will promote the interdisciplinary research of art and design education. More families will have their own family library through interactive television reading. In the future we will stay at home to visit the Digital World Expo, or the Digital Museum. Family-style reading will enter a new era. More families with high quality parenting education can get into the era of interactive primary school education through this platform.
\end{abstract}

Keywords: TV bookstore; Smart TV; Interactive design

DOI: http://dx.doi.org/10.26549/jetm.v2i2.737

\section{Introduction}

$\mathrm{E}$ -bookstore appears as the book content provider into the consumer's vision. Compared to the traditional paper books, it has many advantages, such as low cost, massive storage, convenient search positioning, personalized service, low carbon environmental protection and a variety of services. The operaters not only provide massive reading data, but also provide a new type of terminal reading applications. People read books according to their choice. They accumulated the power of a flower bud. When the opportunity for resurrection comes, the buds will bloom. For example, in the smart TV people find the electronic book on tea culture and open the Tibet buttered tea section. In the virtual sweet tea house Tibetan tea culture enthusiasts can enjoy the scenery of Tibet. In the poetic and artistic scene they sip tea and chat.

The home computer is the reading terminal in the traditional e-bookstore. Because the calculated performance of the home computer is very powerful and the network transmission capability is strong, this method of reading can provide a better reading experience. Today mobile devices can also be read terminals. In addition, there are a large number of mobile reading applications. People provide reading applications by mobile terminals, such as the Amazon Kindle Tablet PC, Hanvon electronic paper book, cloud bookstores, and so on. Compared with home computer, the computing performance and network transmission performance of the kind of mobile terminal is worse. And the screen is relatively small. Although users can read anytime and anywhere by mobile devices, their interactive reading experience is slightly worse.

With the development of technology, household appliances have become more and more intelligent. Television was given high expectations. Now smart TV is being produced. What is smart TV? Smart TV, the same as smartphone, is equipped with a computer operating system. The

Corresponding Author: Xin Xie, yykj0772@126.com 
users can install and uninstall the computer applications by themselves. On the one hand, smart TV must still be a TV or have the function of ordinary television. On the other hand, it has the feature that ordinary TV does not have. The feature is intelligent. The smart TV is more like a smart chatty robot with good interactive performance. The large screen of the high definition can be used in many ways, such as multimedia advertising, reading, games and so on. At present most families have not really used smart TV. One reason is that smart TV lacks a large number of systems and software applications products. These similar products do not have higher performance in mobile terminal of interactive applications. However, "High Mountain does not prevent the white clouds fly, bamboo does not interfere with the flow of water too". Television reading is a kind of entertainment that requires many people to participate in it. Television reading will play an important role in family education in the future. Now some people like to read on their personal computers, while others prefer to read on their mobile phones. As for e-reading, the current competition has been intense between PC and mobile devices. The businesses need to find a breakthrough to expand the business in order to realize "customization of demand" and "entertainment of programs". It is important that we should build a fully open platform in smart TV. We must strive to find a new field of development, and constantly realize the innovation of the program content. Then more family users will be actively involved and experiencing, such as "Reading is happy", "Art education is full of fragrance gladdening the heart and refreshing the mind." etc.

\section{Study on the Design of TV Reading Content}

Flamenco is composed of three elements. It includes cante (singing), toque (guitar playing), baile (dance). Not only Flamenco dancers exercise the body, but also everyone obtain a pleasant mood from flamenco melodies. The content of TV reading needs to be carefully designed. The content can not only open the wisdom of users, but also bring them happy mood. First of all, we need to investigate the behavior and interest of target users. Here, on the basis of a clear user model, a module of tea culture virtual chat is developed by smart TV. People who are proficient in tea ceremony can talk about interesting subjects or programs together. And they may watch opera performances, play games, applaud and shout, and have the feeling of being on the spot. Here are the fragrance of tea, the heat air of the boiling water, the temperature of the teapot, the contemplation of the art. Sometime the user can buy the art blanket online. In the tea-drinking process, the user can directly watering on the blanket to grow green plants.
They will experience the feeling that green flows along with their moods, and so forth.

Here is an example of the application of expanding Interactive design in TV reading. A virtual tea culture house is established. And the animation of user's tea drinking behavior is designed and made. User can experience Chinese tea culture personally on the scene from the animation design process and interactive design. Firstly, a guider guide your tea friends through a street of modern virtual tea culture, as shown in Figure 1. And there are some artworks about virtual tea prop along the street, as shown in Figure 2. When the tea friends walk to the teahouse doorway, the scene of greeting the guest will appear. At the main entrance of the teahouse, the tea friends will meet a lovely teahouse waiter, as shown in Figure 3. When they walked into the teahouse, they can choose the tea room of different theme according to the color map. There are many tea rooms, such as the smoked bean tea room of Taihu Lake, the fragrance tea room of Suzhou, the ginger salt tea of Hunan, the "Xiajun" green tea room of Shushan, the Dongding tea house inTaiwan, Longjing tea house in Hangzhou, Fujian oolong tea room and so on. Different tea rooms were decorated by different waterfall devices of different color. Each teahouse is a kind of digital architectural artwork. For example, in the digital Beijing courtyard building people are tasting the jasmine tea of Zhang Yiyuan. After the tea friends choose the tea room, they go into the tea room. The waiter starts preparing tea, selecting water, heating water and preparing teacups. Then the virtual tea friends will warm the cup, wake up tea, brew tea and enjoy tea, as shown in Figure 4.The graphical user interface of virtual teahouse can be developed from two-dimensional visual sense to three-dimensional visual perception. From the visual perception level, virtual reality technology is introduced into virtual tea room. These technologies can greatly enhance the user experience effect, especially 3D modeling, and the combination of 3D art effect and multi-channel interaction technology. The virtual 3D characters can hear the real human language through the speech recognition technology. Then the virtual $3 \mathrm{D}$ character can make the corresponding action (including voice replies, face expression reply), as shown in Figure 5. The actions may occur in the course of ordering tea. Water ripple effect is made vividly through tactile sensing technology, motion sensing technology and eye tracking technology, as shown in Figure 6. It may be the process of brewing tea in a teacup. As we can see from Fig.7, through the virtual reality technology, the tea user can operate the virtual robot hand to complete the tea pouring movement in the tea ceremony. 


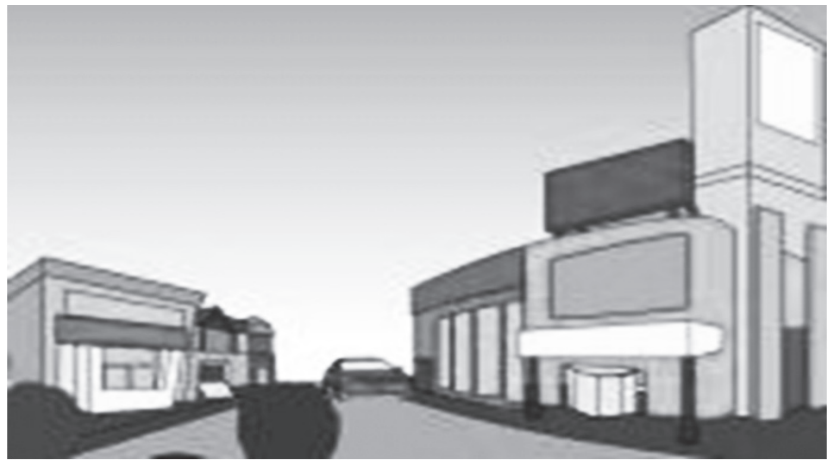

Figure 1. Street of tea culture

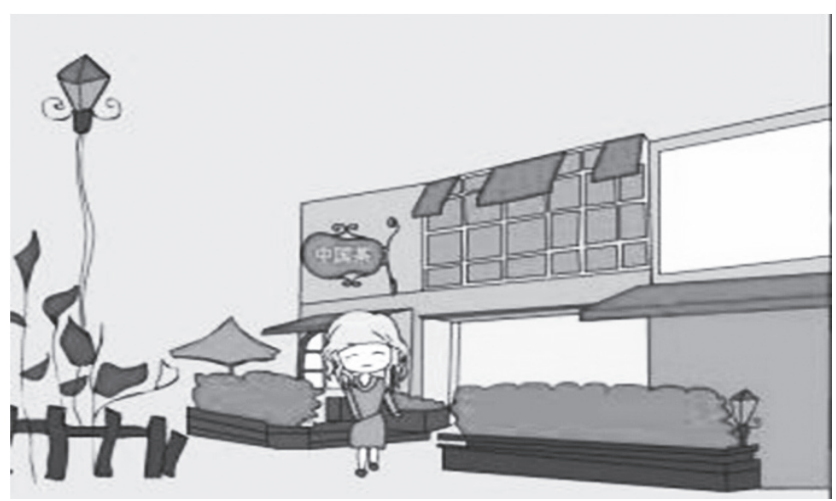

Figure 3. Entrance of the teahouse

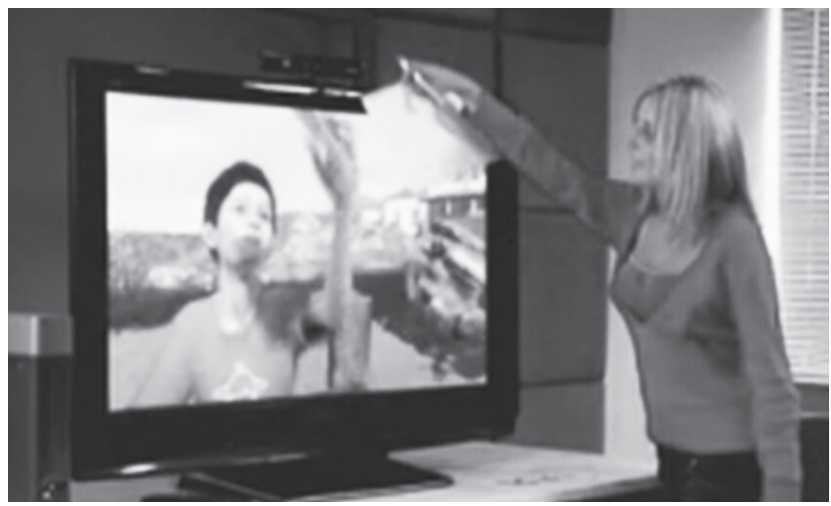

Figure 5. Process of transfer

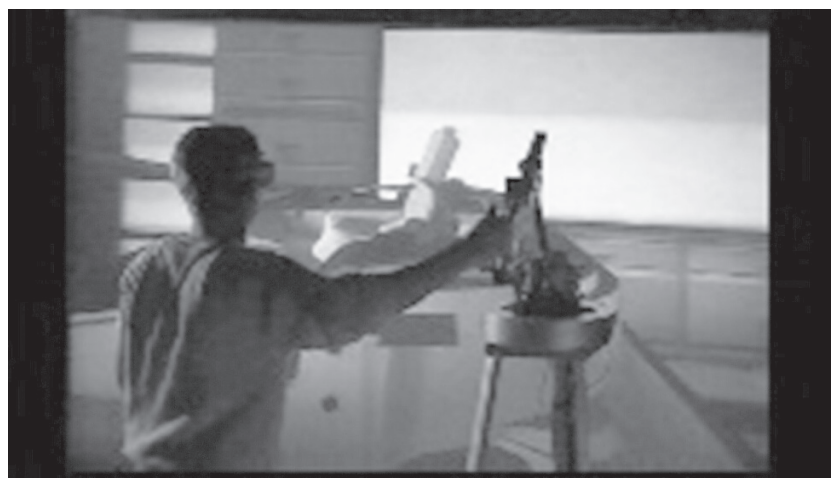

Figure 7. Action of using a lever to refuel

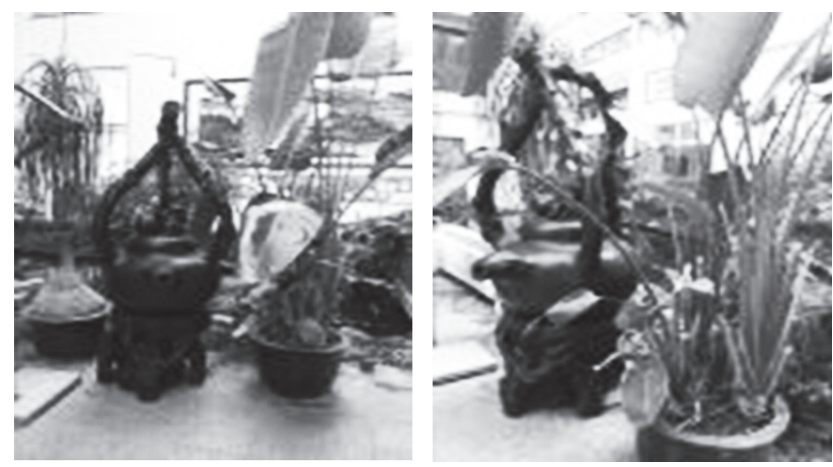

Figure 2. Artworks about tea prop

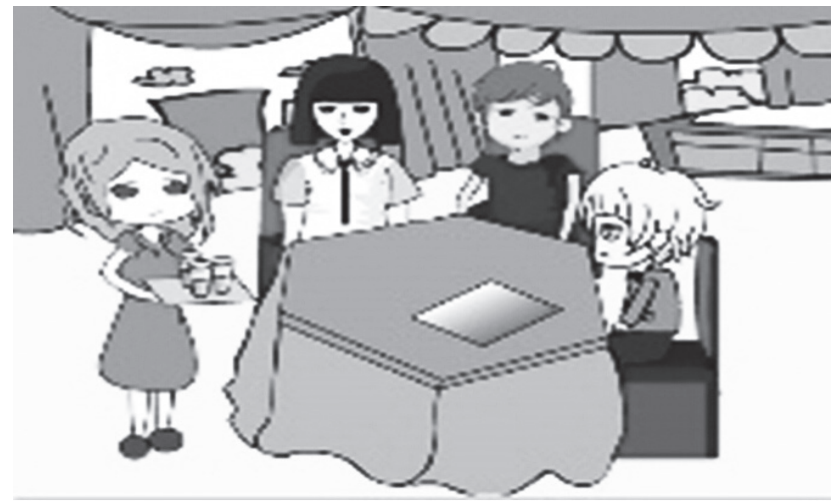

Figure 4. Tea room

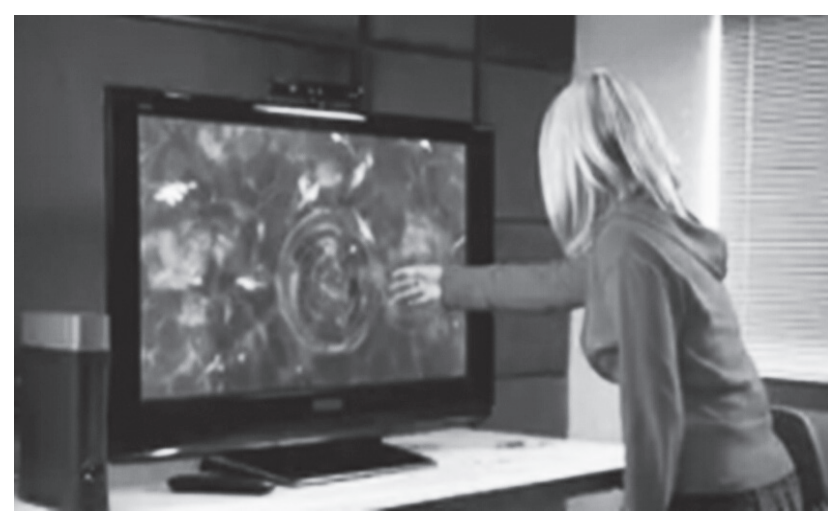

Figure 6. Process of Touching water

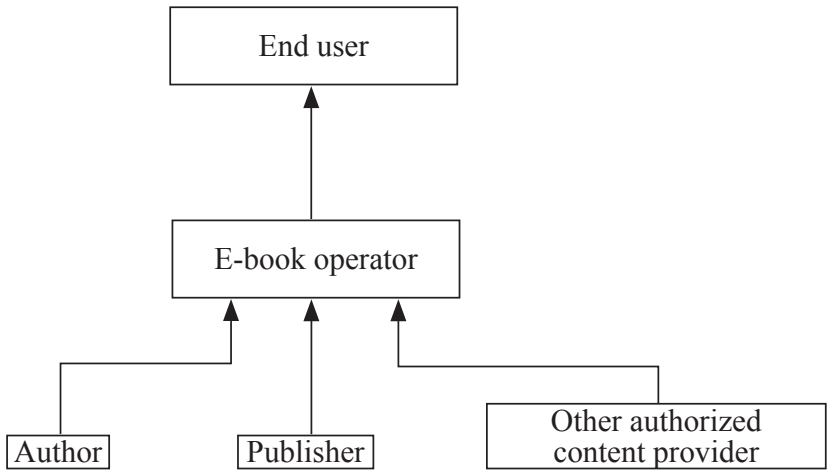

Figure 8. Traditional operation structure 


\section{Imagine of TV Bookstore}

In addition to the innovation of the reading content of the TV bookstore, there is a brand-new design as a product from the TV bookstore. In the future we should develop TV bookstores by smart TV terminals and TV operating entities.

From the point of view of content movement, the implementation model of traditional e-book city is from publishing house to operation entity, and then to end user. The operation structure is shown as Figure 8. The content flows from the downstream to the upper layer in the mode. From the point of view of user perception, the system only occupies the last link of the content movement. The last link is reader device or browser, as shown in Figure 9.

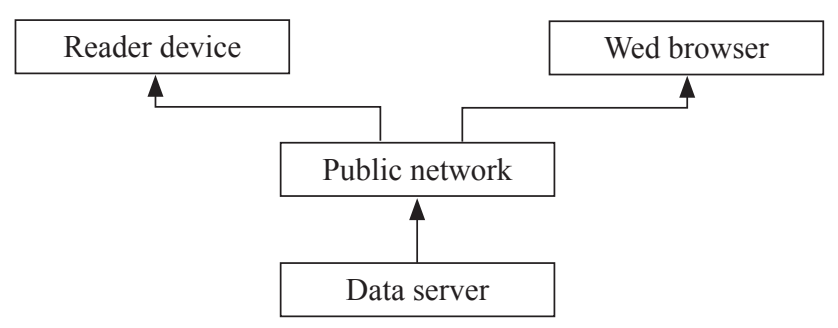

Figure 9. Technology roadmap

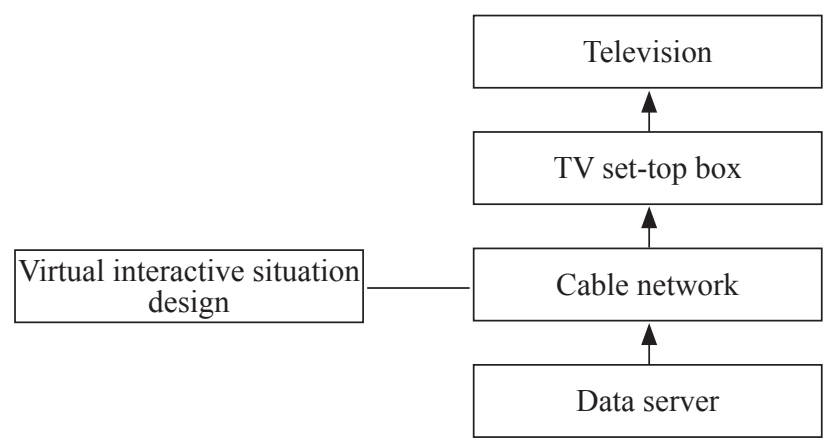

Figure 10. Envisaged reading system

Because there are a lot of pirated software and cracked programs in PC and mobile devices, a lot of content has been stolen and illegally reproduced especially in the last circulation. It is a serious problem for operators. For example, the updated version of the story will be pirated in 15 minutes. Such piracy is too rampant for intellectual property defenders to tolerate. In the Chinese reading site it is a big problem.

In the imagination the content flow direction of the TV bookstore and e-book city is roughly the same. But there are some differences in its operating system, as shown in Figure 10.

In fact, television only is a display device in the high-definition television system. Its video decoding, playback control, VOD system and other functions have been concentrated in the set-top box. We can look upon the set-top boxes as an embedded computer. The operating system and application software is installed in the set-top box. And Google TV is based on the Android operating system.

Imagine: In the TV reading system, the reader can use the smart TV control equipment to select a book or magazine. In the selection process, the set-top boxes send read instructions to the television operating backstage supporter. The backstage supporter can decide whether or not to transmit data according to its permissions and other information. Once the signal is transmitted to the set-top box, its software performs the decoding operation and displays it on the television. You can also apply the control device of smart TV to turn pages or other operations. For example, the average speed of a user's reading is recorded by an intelligent system. This speed refers to the average speed at which people look at books and turn pages. Intelligent software can select the required number of pages according to the reader's needs. Its function provides the speed setup for the user. Firstly people set the resident time of a single page. Then they set the speed of automatic page flipping.

Cable network is not as open as Internet. It is impossible to reproduce content or spread content illegally within the cable television network. The set-top box itself is like a non-public "black box", and therefore it is difficult to intercept electronic reading content from set-top boxes to other networks. Inadvertently the security of content has been protected.

\section{TV Bookstore Research Significance}

Compared with the traditional bookstores, TV bookstores have many advantages.

4.1 The indoor space can be made full use. A personalized atmosphere of indoor reading environment is created.

The visual sensory effects of the TV bookstore can be designed to be similar to the murals which is full of idyllic atmosphere, as shown in Figure 11. A unique e-book cabinet will be built in the TV bookstore of each family. Virtual bookcase will not occupy space. Here users can customize the skin. And they can regularly replace and decorate their own style of virtual bookcase. During the reading process, the TV $3 \mathrm{D}$ interface effect enables the users to feel immersive. Users can experience outdoor reading feeling. It is conducive to physical and mental health. In particular people can stand reading in the front of the hanging television, and enjoy the lightness of reading outdoors. User can move body and neck while reading in the front of the larger screen TV. It will greatly relieve the disease of the cervical spine. 

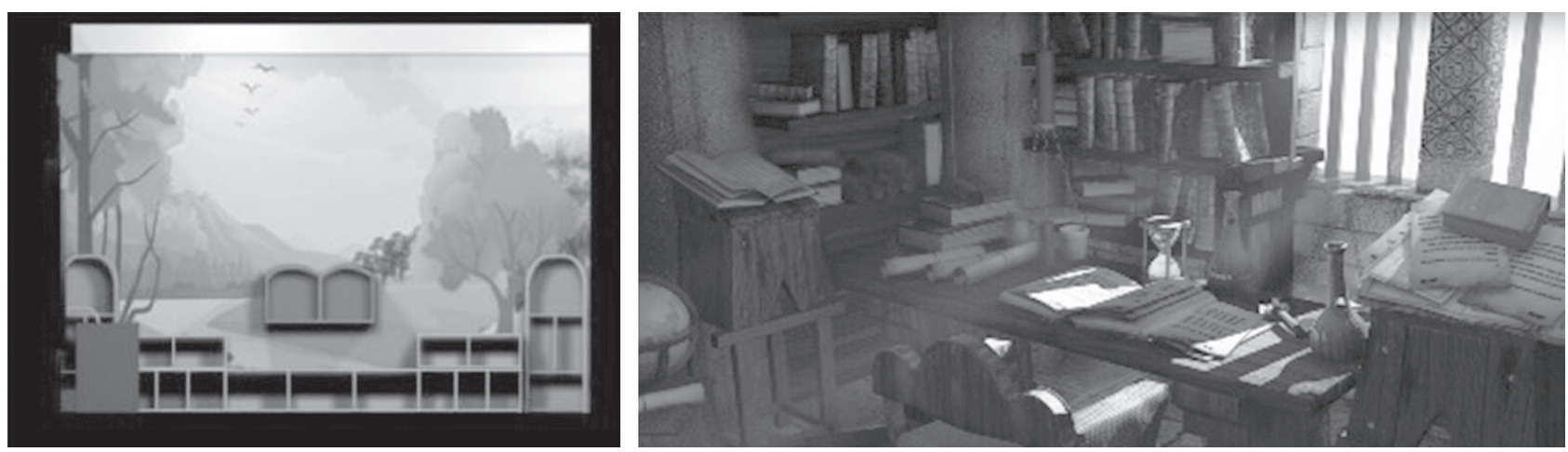

Figure 11. Interface design of TV reading

4.2 A New Way of Life Experience has been Created. And the Economic Value of Television has been Expanded.

There is the characteristic of human-computer interaction in Smart TV. It is wrong for some people to equate the interactive effects of smart TV with video on demand. The real interactive value of television is not limited to video content. It can also include smart reading books, three-dimensional games of touch interaction, the immersion feeling of smell interaction and so on. ${ }^{[1]}$ In fact there are many value-added services to be developed.

The development of smart TV is restricted by many factors. For example, smart TV programs are few. The quality is low .And the update speed is slow. So the TV bookstore can become one of the value-added services during the transition period. It will play an important role in promoting the market. It can inspire audiences and strive for more traditional TV users to participate in future smart multimedia program. TV bookstores will become the one of the most profitable ways of smart TV.

\subsection{Cultural Quality will be Further Improved.}

Through the smart TV bookstore system platform, personalized custom home bookcase will enter all households. In the past, ancient knowledge was well mastered by a small number of people. Today it may appear the "culture into the million family" grand occasion. More and more people master profound knowledge. In the smart TV bookstore culture, users continue to study and accumulate. Constant effort should yield success. ${ }^{[2]}$ People often learn from entertainment by watching TV programs. However, television plots are often different from historical facts, and even do not conform to historical facts. Influenced by "fast food culture", people who have a strong desire for knowledge will give up watching TV shows. Instead, they will choose to turn on the computer. They find the introduction of the story plot or the ending of the story on the Internet. If smart TV has TV bookstore reading function, you will not have to open the computer in the future. It is timely for user to find relevant information about the interested nouns and categories in the situational TV bookstore. In the near future, more parents who want to make family education a source of inspiration for children, will benefit from situational smart TV reading. It will quickly enhance the quality of family education through the smart TV bookstore platform.

\section{Conclusion}

With the intensification of Chinese urbanization process, the application of smart TV bookstore reading is inseparable from the TV book system design. Not only current TV reading is the reading of text and a few pictures, but also it is a reading full of virtual situations that include text, images, interactive animation, audio, video etc. The atmosphere of the TV bookstore will be made up of several virtual chat rooms. These virtual chat rooms have themes. And their human-computer interaction is powerful. The cultural literacy of TV users will be further improved.

Special thanks

This work is the phased result of the project, Research on the animation original brand construction and Internationalization strategy of Beijing (No.SM201511417007).And it is the one of the phased results of the following projects, such as Art education research, Beijing traditional courtyard architectural art of digital experience and creative derivatives design research(No.2017SM201711417005), Teaching reform of WEB technology application development based on MOOCS teaching mode (No.JJ2016Y067), Interactive media module training project development (No. JJ2015Y090), Weight analysis of main factors affecting imbibition efficiency (No. 11107JA1604).

\section{References}

[1] Chunmei Gan, Wang Weijun, Academic Blogs' Willingness to Use: Interaction, Immersion and Satisfaction[J]. Information Science, 2015, (03). (in Chinese)

[2] Jiu Liang, Where road painless[M].Chong Qing University Press, 2016, pp.191-192. (in Chinese) 